\title{
The Mu2e Tracker
}

\section{G. Pezzullo*t}

Yale University, Physics Department, 217 Prospect Street, New Haven (CT), USA

E-mail: g.pezzullo@yale.edu

The Mu2e experiment will search for neutrinoless conversion of muons into electrons In the field of an aluminum nucleus. The signature of this process is an electron with energy nearly equal to the muon mass. Precise and robust measurement of the outgoing electron momentum, combined with other background rejection methods, is essential to the experiment. We rely on a low-mass straw tube tracker to achieve these goals. The tracking system must operate in a vacuum and a 1 Tesla magnetic field. We have chosen to use about 20,000 thin-wall Mylar straws held under tension to avoid the need for support within the active volume. In addition to measuring the distance from the wire by drift time, subnanosecond measurement of signal propagation time will be used to measure position along the wire. The charge will be measured using ADCs to provide particle identification capability.

The 39th International Conference on High Energy Physics (ICHEP2018)

4-11 July, 2018

Seoul, Korea

*Speaker.

${ }^{\dagger}$ on behalf of the Mu2e Tracker group. 


\section{Introduction}

Mu2e is an experiment, based at the Fermi National Laboratory, that will search for the coherent conversion of a $\mu^{-}$into an $e^{-}$in the field of an $\mathrm{Al}$ nucleus. This process is not allowed by the Standard Model (SM) because it would violate the conservation of the Lepton family number. In addition to that, even if we include the neutrino oscillation in the SM, the rate at which this process is predicted is inaccessible by the current facilities $\left(\sim 10^{-52}\right)$. The interesting part is that many models of Physics beyond the SM predict this process with a rate accessible by Mu2e [1]. The experimental signature of the $\mu \mathrm{N} \rightarrow e \mathrm{~N}$ is quite clean; it consists of a monochromatic $e^{-}$with an energy slightly below the $\mu$ rest mass, $\mathrm{E}=104.97 \mathrm{MeV}$ because we need to take into account the contributions from the binding energy and the nucleus recoil [3]. The Mu2e experimental apparatus consists of three main superconducting solenoids: the first two, named Production and Transport solenoids respectively, are aimed to create a low-momentum muon beam $(<\mathrm{p}>\sim 60 \mathrm{MeV} / \mathrm{c})$, while the third one houses the Al Stopping Target, where the muons are stopped, and the Detector system [2]. The "core" of the detector system is the Tracking chamber that is described in the next sections.

\section{Design}

The tracker design was optimized in order to maximize the acceptance for the conversion electrons and, at the same time, minimize the background contribution coming from the electrons from the muon decay in orbit processes. The main features of this background are: (i) the endpoint of the momentum spectrum coincides with the Conversion electron energy; (ii) about $10^{-16}$ of its spectrum is within the last $1 \mathrm{MeV}$ [3]. This is why a momentum resolution better than few hundreds of $\mathrm{keV} / \mathrm{c}$ is important for the success of the experiment. The tracker consists of 36 tacking planes equally spaced along $3.2 \mathrm{~m}$ with straws transverse to the beam line. The tracking plane is a modularized structure made out of 6 panels. A single panel is made out of 96 staggered straw-tubes with the following specs: (i) $5 \mathrm{~mm}$ diameter; (ii) $2 \times 6.25 \mu \mathrm{m}$ Mylar walls Au and Al coated; (iii) 25 Âţm Au-plated W sense wire. The straw-tubes are filled with an $\mathrm{Ar} / \mathrm{CO}_{2}$ gas mixture (80:20) at $1 \mathrm{~atm}$ differential pressure and they have a length varying from 44 up to $114 \mathrm{~cm}$. Figure 1 shows a schematic representation of the tracker. The sag effect, due to the relaxation of the straws, has

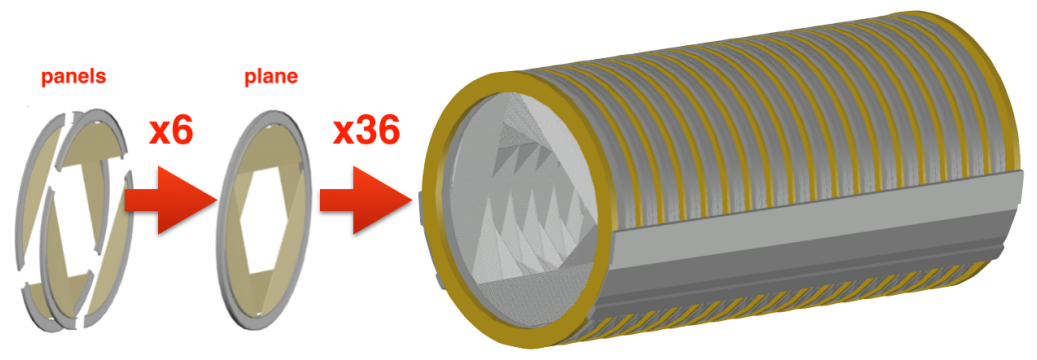

Figure 1: Schematic view of a straw-tube panel with electronics front-end and radout-controller board installed.

been deeply studied during the last years to confirm that a sag $\leq 200 \mu \mathrm{m}$ can be achieved with this 
design [6]. Another important requirement for the tracker is related to the vacuum leak. There are $\sim 20 \mathrm{k}$ straws that collectively must leak less than $6 \mathrm{sccm}$. To qualify all the straw-tubes needed for the assembly, we developed custom chambers that can measure the leak rate of multiple straws at the same time.

\section{Electronics}

The readout controller board (DRAC) is housed in the outer part of each panel together with the straw-tube front-end electronics. Monte Carlo simulation studies showed that the expected peak dose in electronics is about $150 \mathrm{krad}$. An irradiation campaign was performed in order to qualify all the major components of the DRAC: the FPGA PolarFire from Microsemi ${ }^{1}$, few commercial DC/DC converters under study and the Versatile optical transceiver from CERN [5]. Figure 2 shows the prototype of a panel with the front-end electronics installed.

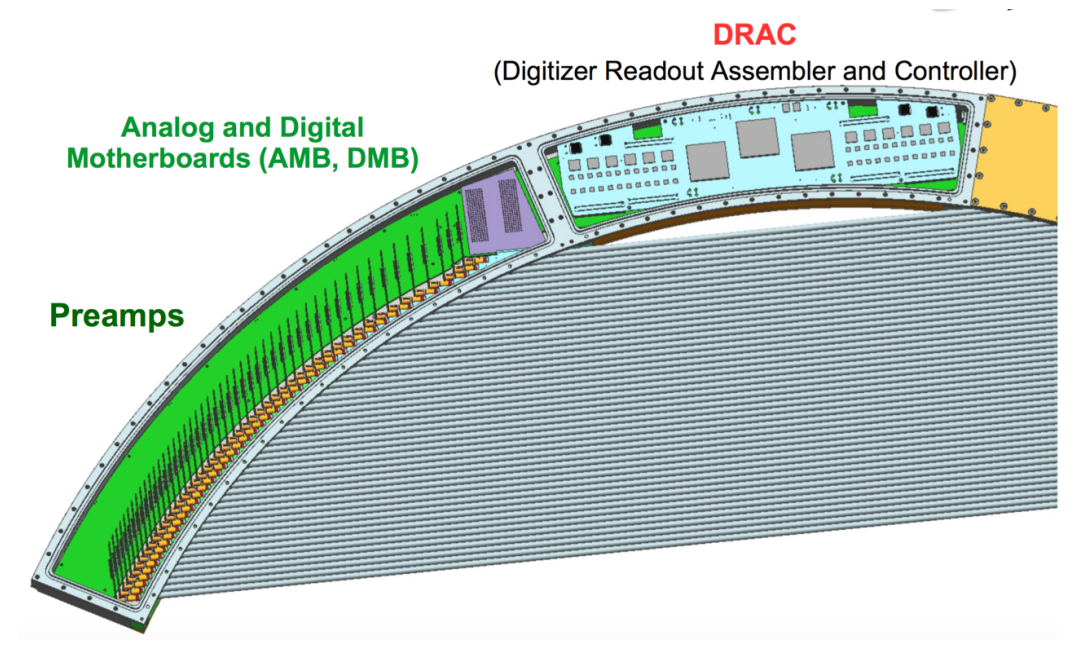

Figure 2: Prototype of a strawtube panel with electronics front-end and radout-controller board installed.

\section{Prototype results}

A small panel prototype with 8 straws was built at Lawrence Berkeley Laboratories to measure the performance using cosmic rays and radioactive sources [7]. The straw resolution was measured for each of the 8 straws using cosmic rays. The Mu2e official Monte Carlo simulation implements a detailed model of physical and electronics responses to GEANT4 [4] energy deposition. This model includes all the chain electron Clusters $\rightarrow$ Drift $\rightarrow$ Current pulse $\rightarrow$ Voltage waveform $\rightarrow$ Digitization. Figure 3 shows the distribution of the drift distance residual (blue line) of the cosmic ray data. The transverse resolution is found to have a full-width half max of $283 \mu \mathrm{m}$.

\footnotetext{
${ }^{1}$ Datasheet, https://www.microsemi.com/product-directory/fpgas/3854-polarfire-fpgas (last access: 2018-10-30
} 


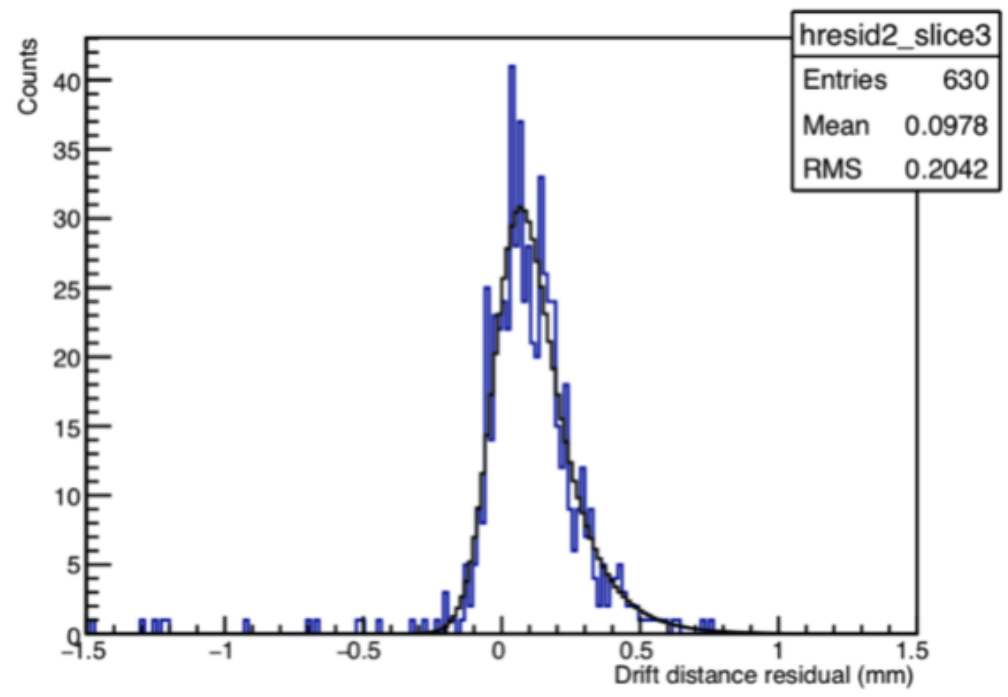

Figure 3: Distribution of the drift distance residual from cosmic data (blue line) fit to a function that includes both a Gaussian smearing component as well as a exponential component that includes geometric effects.

\section{Summary}

This paper presented the Mu2e tracker design, including experimental results from a small detector prototype. The Mu2e tracker has been designed to provide an accurate reconstruction of the $\mu \mathrm{N} \rightarrow e \mathrm{~N}$ signal and operate in a harsh environment where is expected a non-negligible amount of dose. The R\&D phase of the tracker is mature and the panel assembly has started in summer 2018.

\section{Acknowledgements}

We are grateful for the vital contributions of the Fermilab staff and the technical staff of the participating institutions.

\section{References}

[1] Kitano, R. et al, Detailed calculation of lepton flavor violating muon electron conversion rate for various nuclei, Phys. Rev., D66, 2002

[2] Bartoszek, L. et al, Mu2e Technical Design Report, arXiv: 1501.05241 [physics.ins-det], 2014

[3] Bernstein, Robert H. et al, Charged Lepton Flavor Violation: An Experimenter's Guide, Phys. Rep., 532, 2, 27-64, 2013

[4] Agostinelli, S. et al, Geant4 - a simulation toolkit, NIM A, 506, 250-303, 2003

[5] Olantera, L. et al, Versatile transceiver production and quality assurance, JINST, 12, 01, C01097, 2017

[6] Bono, J.S. et al, The stress relaxation (creep) rate of Mu2e straw tubes, NIM A, 902, 95-102, 2018

[7] Brown, D. et al, An 8-Straw Prototype Tracker for Mu2e, 2016, https://indico.cern.ch/event/432527/contributions/1072368/contribution.pdf, (last access: 2018-10-30) 